\title{
RELATIONSHIP OF METHYL MERCAPTAN AND HYDROGEN SULFIDE LEVELS WITH TANNERELLA FORSYTHIA QUANTITY IN PERIODONTITIS PATIENTS WITH HALITOSIS AND DIABETES MELLITUS
}

\begin{abstract}
ADINDA R FITRIA $^{1}$, YUNIARTI SOEROSO ${ }^{2 *}$, NATALINA², DICKY L TAHAPARY ${ }^{3,4}$, BOY M BACHTIAR ${ }^{5}$
${ }^{1}$ Periodontics Residency Program, Faculty of Dentistry, Universitas Indonesia, Jakarta, Indonesia. ${ }^{2}$ Department of Periodontics, Faculty of Dentistry, Universitas Indonesia, Jakarta, Indonesia. ${ }^{3}$ Division of Endocrinology and Metabolism, Department of Internal Medicine,

Dr. Cipto Mangunkusumo National Referral Hospital, Faculty of Medicine Universitas Indonesia, Jakarta, Indonesia. ${ }^{4}$ Metabolic, Cardiovascular and Aging Cluster, The Indonesian Medical Education and Research Institute, Faculty of Medicine Universitas Indonesia, Jakarta, Indonesia. ${ }^{5}$ Department of Oral Biology, Faculty of Dentistry, Universitas Indonesia, Jakarta, Indonesia. Email: yuniarti@ui.ac.id
\end{abstract}

Received: 25 September 2019, Revised and Accepted: 29 June 2020

\section{ABSTRACT}

Objective: Halitosis may be caused by several factors, including various types of food, periodontal diseases, layer of tongue bacteria, and systemic disorders such as diabetes mellitus (DM), which is a chronic disease that affects the health of periodontal tissue. The present study aimed to assess the association between the quantity of Tannerella forsythia bacteria and the levels of methyl mercaptan and hydrogen sulfide in periodontitis patients with type 2 DM (T2DM).

Methods: Gingival crevicular fluids (GCF) were collected from 20 patients who were divided into those with periodontitis and who were normoglycemic $(n=8)$; those with periodontitis and T2DM $(n=8)$; and healthy controls $(n=4)$. The patients underwent intraoral periodontal tissue examination, including pocket depth, attachment loss, plaque index, calculus index, and papilla bleeding index. The quantity of T. forsythia bacteria was evaluated using quantitative real-time reverse transcription-polymerase chain reaction. The relationship between the number of $T$. forsythia bacteria and the levels of methyl mercaptan and hydrogen sulfide in the patients was analyzed by Spearman's correlative tests.

Results: There is a weak and non-significant correlation $(p>0.05)$ between the levels of methyl mercaptan and hydrogen sulfide and the quantity of T. forsythia in the GCF and tongue coating of periodontitis patients with halitosis regardless of the presence of T2DM.

Conclusion: This study suggests no significant relationship between the levels of methyl mercaptan and hydrogen sulfide and the quantity of T. forsythia in periodontitis patients with halitosis and DM.

Keywords: Methyl mercaptan, Hydrogen sulfide, Tannerella forsythia, Periodontitis, Halitosis, Diabetes mellitus.

(c) 2020 The Authors. Published by Innovare Academic Sciences Pvt Ltd. This is an open access article under the CC BY license (http://creativecommons. org/licenses/by/4. 0/) DOI: http://dx.doi.org/10.22159/ijap.2020.v12s2.0P-59

\section{INTRODUCTION}

Halitosis, also known as fetor oris, oral bad breath, or simply bad breath, influences the quality of a person's social interactions and his/ her level of confidence. The main factors causing halitosis are local factors such as plaque, calculus, periodontal disease, dental caries, xerostomia, tongue coating (TC), tobacco use, and oral cavity infections. Other factors include several systemic factors such as diabetes, upper respiratory infections, and types of food [1].

Malodor is associated with the degradation of sulfur-containing amino acids (methionine, cysteine, and cystine) by anaerobic Gram-negative bacteria present in the oral cavity, including Porphyromonas gingivalis, Fusobacterium nucleatum, Prevotella intermedia, Tannerella forsythia, and $P$. endodontalis. Volatile sulfur compounds (VSC), such as hydrogen sulfide $\left(\mathrm{H}_{2} \mathrm{~S}\right)$, methyl mercaptan $\left(\mathrm{CH}_{3} \mathrm{SH}\right)$, and dimethyl sulfide $\left[\left(\mathrm{CH}_{3}\right)_{2} \mathrm{~S}\right]$, are produced as metabolic products of degradation [2]. Research has demonstrated that VSCs are major contributors to bad breath. Hydrogen sulfide, methyl mercaptan, and to a lesser extent, $\left(\mathrm{CH}_{3}\right)_{2} \mathrm{~S}$, represent $90 \%$ of the VSCs in bad breath [3].

Halitosis is divided into two conditions - delusional (pseudohalitosis and halitophobia) and original halitosis. Original halitosis is further divided into two subconditions - physiological and pathological halitosis. Pathological halitosis can be oral or extraoral [4], with oral pathologies that include conditions such as tongue biofilm, bad oral hygiene, and periodontal disease. Periodontal disease is a polymicrobial immune-inflammatory disease that can cause destruction of periodontal ligaments and adjacent supporting alveolar bones. Subgingival plaques comprise more than 700 bacterial species, and some of these species are involved in the early stages of periodontal diseases [5]. The primary etiological factor of most periodontal diseases is bacterial plaque comprising pathogenic microflora growing in the periodontal pocket and teeth. Some bacteria are able to attach to and colonize the tooth surface, thus establishing a place for colonization of other microorganisms. Basically, periodontitis begins with the inflammation of the gingiva in response to bacteria, which represents a pathogenic shift in bacterial flora around the teeth [3].

Diabetes mellitus (DM) is a metabolic disease that has the characteristics of hyperglycemia and occurs due to abnormalities in insulin secretion, insulin action, or both. DM is characterized by chronic hyperglycemia due to both relative and absolute insulin deficiency [6]. The triad of symptoms in people with DM is called polydipsia, polyuria, and polyphagia. These symptoms are direct results of hyperglycemia and the subsequent osmotic imbalance [7].

The periodontal sites of individuals with critical glycemic control are associated with an increased frequency of T. forsythia and the four orange complex species studied here compared with those of patients with better controlled glycemia [8]. T. forsythia is a periodontopathogenic bacterium belonging to the red complex group that includes the main 
causative agents of periodontal diseases. It has been detected in significantly higher quantities in obese patients (both diabetic and non-diabetic) compared with non-obese non-diabetic controls [9]. The present study aimed to analyze the relationships between the levels of methyl mercaptan and hydrogen sulfide and the quantities of T. forsythia bacteria in periodontitis patients with halitosis and DM.

\section{METHODS}

The study population comprised patients at the clinic for the Periodontics Section of the University of Indonesia Dental and Oral Hospital, and IMERI, the Faculty of Medicine, University of Indonesia, starting from June 2019 to July 2019. Research at the laboratory was conducted at the Oral Biology Laboratory, Faculty of Dentistry, University of Indonesia.

This study was implemented with a cross-sectional design. Subjects were divided into three groups, which are control group $(\mathrm{n}=4)$, periodontitis group $(n=8)$, and periodontitis with type 2 DM (T2DM) group $(n=8)$. All groups were selected from the existing patient population, and then examined to determine whether they had periodontitis with halitosis or whether they had periodontitis with halitosis accompanied by DM. All the research patients signed informed consent forms to demonstrate their willingness to participate in the study. The research process initiated with a brief history, followed by intraoral examination and sample collection. The inclusion criteria were as follows: Adults (17-50 years); with no history of aggressive periodontitis; with a history of DM, especially T2DM; with an HbA1C value; ( $\geq 6.5 \%$ ); and with a history of halitosis (both subjective and objective). The exclusion criteria were as follows: A history of periodontitis with systemic diseases other than DM; receiving periodontal treatment within the previous 3 months before the examination; smoked within $<24 \mathrm{~h}$ before the examination; used antibiotics within the previous month before the examination; used fragrances; consumed foods that caused bad breath; and underwent fasting.

Clinical examinations were performed to evaluate periodontal tissue conditions in the form of pocket depth using the UNC 15 Periodontal Probe (Osung Mnd, Korea) to collect data on plaque index (PI), calculus index $(\mathrm{KI})$, and papilla bleeding index.

The levels of methyl mercaptan and hydrogen sulfide were measured using OralChroma (Morita, Japan) in 2014, following the manufacturer's instructions. Briefly, the patients were asked to close their mouth tightly and not speak for $3 \mathrm{~min}$. Gas was collected with a 1-mL syringe by inserting its body into the tip of the oral cavity. The patients were asked not to blow or suck into the syringe when gas was obtained from the oral cavity. A 27-gauge needle was attached to the body of the syringe, and its gas contents were injected into a port in OralChroma. While OralChroma was measuring the levels of methyl mercaptan and hydrogen sulfide, an intraoral examination was performed. Oral hygiene checks, pocket examinations, and TC scores were recorded. The periodontal pocket comprises the absolute sac, without gingival recession, and is measured from the gingival margin to the base of the sulcus. Organoleptic tests were also performed by the same operator for each patient.

Microbial samples were collected from gingival crevicular fluid (GCF) using a sterilized no. 30 paper point (30 s in the deepest pocket) and from TC using sterile cotton swabs. The surface to be sample was first air-dried and wiped with a cotton roll before the gingival sulcus fluid was sampled. The paper point was carefully inserted into the gingival sulcus for $30 \mathrm{~s}$, ensuring that bleeding from the gingiva is prevented. During sampling, the surrounding area was dried and all efforts were taken to avoid bleeding. The samples were placed in a sterile $1.5-\mathrm{mL}$ microcentrifuge tube using Ringer Solution as a transfer medium. Twenty samples collected from GCF and tongues coating were frozen at $-20^{\circ} \mathrm{C}$ and transferred to the Oral Biology Laboratory for analysis.

Total DNA was extracted using the GENEzolTM reagent following the manufacturer's instructions. DNA concentration was measured by the
Qubit ${ }^{\circledR}$ 3.0 Fluorometer (ThermoFisher Scientific, USA). Quantitative real-time reverse transcription-polymerase chain reaction (qRT-PCR) was performed on the ABI StepOnePlus RT-PCR System with the SYBR Green PCR master (Applied Biosystems, USA) following the manufacturer's instructions. DNA samples were amplified with T. forsythia primers (Table 1) [10]. The thermal cycling conditions were as follows: Pre-denaturation at $95^{\circ} \mathrm{C}$ for $5 \mathrm{~min}$, followed by 40 amplification cycles comprising $95^{\circ} \mathrm{C}$ for $10 \mathrm{~s}, 60^{\circ} \mathrm{C}$ for $30 \mathrm{~s}$, and $72^{\circ} \mathrm{C}$ for $30 \mathrm{~s}$. The melting curve profile was set at $95^{\circ} \mathrm{C}$ for $15 \mathrm{~s}, 60^{\circ} \mathrm{C}$ for $60 \mathrm{~s}$, and $95^{\circ} \mathrm{C}$ for $15 \mathrm{~s}$. This study was approved by the Ethical Committee of Dental Research (KEPKG), Faculty of Dentistry, Universitas Indonesia (protocol number 090460419).

\section{Statistical analysis}

Data were analyzed using descriptive statistics, and hypothesis testing statistics was used to test whether there is a relationship between methyl mercaptan and hydrogen sulfide levels and the number of T. forsythia bacteria in periodontitis patients with halitosis complaints accompanied by DM. Data were analyzed using the SPSS (SPSS, Chicago, USA) software program. To determine the existence and direction of these relationships, Spearman's correlative test was performed $(\mathrm{p}>0.05)$.

\section{RESULTS AND DISCUSSION}

Based on Table 2, the levels of hydrogen sulfide between periodontitis patients and periodontitis patients with DM differed significantly ( $p=0.011$ ). The proportion of T. forsythia in the GCF and TC and the quantities of methyl mercaptan between periodontitis patients and periodontitis patients with DM do not differ significantly.

The levels of T. forsythia in the GCF of periodontitis patients and periodontitis patients with DM did not differ significantly. T. forsythia is a Gram-negative anaerobic bacteria that are considered the main periodontal pathogen [11,12]. Farias et al. reported that these bacteria are commonly found in deep pockets where the oxygen partial pressure is relatively low [11]. T. forsythia, P. gingivalis, and Treponema denticola form a "red complex" of bacterial species strongly associated with severe chronic periodontitis [12].

Farias et al. evaluated the clinical and microbiological data of patients with chronic periodontitis and found elevated amounts of $T$. forsythia in pockets deeper than $8 \mathrm{~mm}$. Moreover, the quantity of T. forsythia is elevated following increased interactions with several other bacteria, such as $P$. gingivalis and T. denticola [11]. Several independent studies on different populations worldwide have demonstrated a great number of T. forsythia in the subgingival plaques of patients with periodontitis [12].

The proportion of T. forsythia on the TCs of periodontitis patients and periodontitis patients with DM did not differ significantly. A recent observational study on patients aged $t 40$ years demonstrated that periodontitis is more prevalent among individuals with diabetes than in non-diabetic patients; this association holds true regardless of patients' sex and age [13].

The specific mechanism connecting DM and periodontal disease has not been fully elucidated. Several reports have suggested that DM alters the subgingival bacterial community through changes in the substrate that offers a favorable microenvironment for the growth of pathogens [13]. Patients with DM often have decreased salivary flow

Table 1: Tannerella forsythia primer sequences

\begin{tabular}{lll}
\hline Primer name & Sequences & References \\
\hline Tannerella & Forward: 5'-GGG TGA GTA ACG CGT & {$[10]$} \\
forsythia & ATG TAA CCT-3' & \\
& Reverse: 5'-GCC CAT CCG CAA CCA & \\
& ATA AA-3' \\
\hline
\end{tabular}


Table 2: Comparison of Tannerella forsythia in GCF and tongue coating, proportion of methyl mercaptan, and hydrogen sulfide between periodontitis patients and periodontitis patients with diabetes mellitus

\begin{tabular}{lll}
\hline & Median (Min-Max) & p value \\
\cline { 2 - 3 } & Periodontitis (n=8) & Periodontitis with DM (n=8) \\
\hline Tannerella forsythia in GCF & $33.88(3.75-379.18)$ & $403.99(13.24-4242.65)$ \\
Tannerella forsythia in tongue coating & $536.51(103.44-1830.97)$ & $362.61(10.88-2747.65)$ \\
Methyl mercaptan & $0.49(0.01-8.88)$ & $1.04(0.28-2.42)$ \\
Hydrogen sulfide & $1.39(0.28-4.65)$ & $0.00(0.00-10.05)$ \\
\hline
\end{tabular}

Comparison significance at $\mathrm{p}<0.05$. GCF: Gingival crevicular fluid

Table 3: Correlations between the proportion of Tannerella forsythia in the GCF and TC of periodontitis patients and periodontitis patients with diabetes mellitus

\begin{tabular}{|c|c|c|c|c|}
\hline & \multicolumn{2}{|c|}{ r values of Tannerella forsythia in GCF } & \multicolumn{2}{|c|}{ r values of Tannerella forsythia in TC } \\
\hline & Periodontitis & Periodontitis with DM & Periodontitis & Periodontitis with DM \\
\hline Methyl mercaptan [p] & $0.060[0.887]$ & $-0.024[0.955]$ & $-0.277[0.506]$ & $0.382[0.351]$ \\
\hline Hydrogen sulfide [p] & $0.214[0.610]$ & $0.214[0.610]$ & $1.00[0]$ & $-0.071[0.867]$ \\
\hline
\end{tabular}

GCF: Gingival crevicular fluid, TC: Tongue coating

and high salivary viscosity, which reduces the mouth's cleaning capacity as well as the action of salivary antimicrobial factors. These conditions facilitate the retention of exfoliating mucous cells and the proliferation of microorganisms, especially on tongue surfaces. High retention of cells on the tongue surface facilitates the deposition and proliferation of microorganisms [14].

GCF, TC correlations are considered significant at $\mathrm{p}<0.05$. Based on our Spearman's correlative tests, the proportion of T. forsythia on the tongues of periodontitis patients is perfectly correlated with the levels of hydrogen sulfide. The remaining tests revealed no other significant correlations between the quantity of $T$. forsythia and the levels of methyl mercaptan and hydrogen sulfide. There is negative correlation between the number of $T$. forsythia in the GCF and TC both on methyl mercaptan and hydrogen sulfide, whereas the positive correlation between the levels of methyl mercaptan and hydrogen sulfide and the number of T. forsythia in the GCF and the tongue lining.

Table 3 shows that the number of T. forsythia in TCs is significantly correlated with the levels of hydrogen sulfide. This indicates that the proportion of T. forsythia in the TC leads to increased levels of hydrogen sulfide in periodontitis patients. In addition, Table 3 shows no other significant result. This can be explained by the presence of factors other than T. forsythia that result in increased levels of methyl mercaptan in both the GCF and TC of periodontitis patients or increased levels of hydrogen sulfide in the GCF of periodontitis patients.

Table 3 also shows that the number of T. forsythia in the GCF and TC of periodontitis patients with DM is correlated but not significant with the levels of both methyl mercaptan and hydrogen sulfide. This is because halitosis patients with DM have different patterns/types of gases that cause halitosis. Usually, patients with DM have bad breath caused by pathologic halitosis that tends to lower the levels of methyl mercaptan and hydrogen sulfide. This may explain why the levels of methyl mercaptan and hydrogen sulfide were not significantly correlated with the proportion of $T$. forsythia, although the absolute proportion of T. forsythia is high. Extraoral halitosis may originate from the respiratory or other system [4]. Systemic diseases or the disease process affect the host defense system, thus acting as a risk factor for gingivitis and periodontitis. Therefore, some host responses can be associated with increased incidence and severity of periodontitis in diabetics [15].

\section{CONCLUSION}

The proportion of T. forsythia is higher on the TC both in periodontitis patients and periodontitis patients with DM; however, the proportion of T. forsythia in the GCF and TC of periodontitis patients does not differ significantly from those of periodontitis patients with DM. The levels of methyl mercaptan and hydrogen sulfide are not significantly correlated with the quantity of T. forsythia in the GCF and TC. Further studies are needed to confirm the results of this study.

\section{ACKNOWLEDGMENT}

This project was supported by a grant from HIBAH PITA UI 2019, University of Indonesia.

\section{CONFLICTS OF INTEREST STATEMENT}

The authors declare that they have no conflicts of interest.

\section{REFERENCES}

1. Alasqah M, Khan S, Elqomsan MA, Gufran K, Kola Z, Hamza MO. Assessment of halitosis using the organoleptic method and volatile sulfur compounds monitoring. J Dent Res Rev 2016;3:94-8.

2. Calil CM, Oliveira GM, Cogo K, Pereira AC, Marcondes FK, Groppo FC. Effects of stress hormones on the production of volatile sulfur compounds by periodontopathogenic bacteria. Braz Oral Res 2014;28:1-8.

3. De Geest S, Laleman I, Teughels W, Dekeyser C, Quirynen M. Periodontal diseases as a source of halitosis : A review of the evidence and treatment approaches for dentists and dental hygienists. Periodontol 2000 2016;71:213-27.

4. Bicak DA. A current approach to halitosis and oral malodor a mini review. Open Dent J 2018;12:322.

5. Suzuki N, Yoneda M, Hirofuji T. Mixed red-complex bacterial infection in periodontitis. Int J Dent 2013;2013:587279.

6. Ermawati T, Periodonsia B, Kedokteran F. Universitas G. Periodontitis dan diabetes melitus. JKG Unej 2013;9:152-154

7. Soskolne WA, Klinger A. The relationship between periodontal diseases and diabetes: An overview. Ann Periodontol 2001;6:91-8.

8. Miranda TS, Feres M, Retamal-Valdes B, Perez-Chaparro PJ, Maciel SS, Duarte PM. Influence of glycemic control on the levels of subgingival periodontal pathogens in patients with generalized chronic periodontitis and Type 2 diabetes. J Appl Oral Sci 2017;25:82-9.

9. Al-Rawi N, Al-Marzooq F. The relation between periodontopathogenic bacterial levels and resistin in the saliva of obese Type 2 diabetic patients. J Diabetes Res 2017;2017:2643079.

10. Soeroso Y, Akase T, Sunarto H, Kemal Y, Salim R, Octavia M, et al. The risk reduction of recurrent periodontal pathogens of local application minocycline $\mathrm{HCL}_{2} \%$ gel, used as an adjunct to scaling and root planing for chronic periodontitis treatment. Ther Clin Risk Manag 2017;13:307-314.

11. Soeroso Y, Kemal Y, Widodo A, Bachtiar B, Pontoh D. Relationship between the quantitative measurement of Tannerella forsythia on dental plaque and its relationship with the periodontal status of patients with 
coronary heart disease. J Int Dent Med Res 2018;11:1054-60.

12. Jusko M, Potempa J, Karim AY, Ksiazek M, Riesbeck K, Garred P, et al. A metalloproteinase karilysin present in the majority of Tannerella forsythia isolates inhibits all pathways of the complement system. J Immunol 2012;188:2338-49.

13. Liccardo D, Cannavo A, Spagnuolo G, Ferrara N, Cittadini A, Rengo C, et al. Periodontal disease: A risk factor for diabetes and cardiovascular disease. Int J Mol Sci 2019;20:1-14.

14. Negrato CA, Tarzia O. Buccal alterations in diabetes mellitus. Diabetol Metab Syndr 2010;2:3.

15. Ryan ME, Carnu O, Kamer A. The influence of diabetes on the periodontal tissues. J Am Dent Assoc 2003;134:34S-40S. 